\title{
Image of the month: Severe Raynaud's phenomenon and Sjögren's syndrome with ferocious gangrene change and auto-amputation
}

\author{
Authors: Pin-Hsiang Huang, ${ }^{A}$ Hsien-Tzung Liao ${ }^{B}$ and Chang-Youh Tsai ${ }^{C}$
}

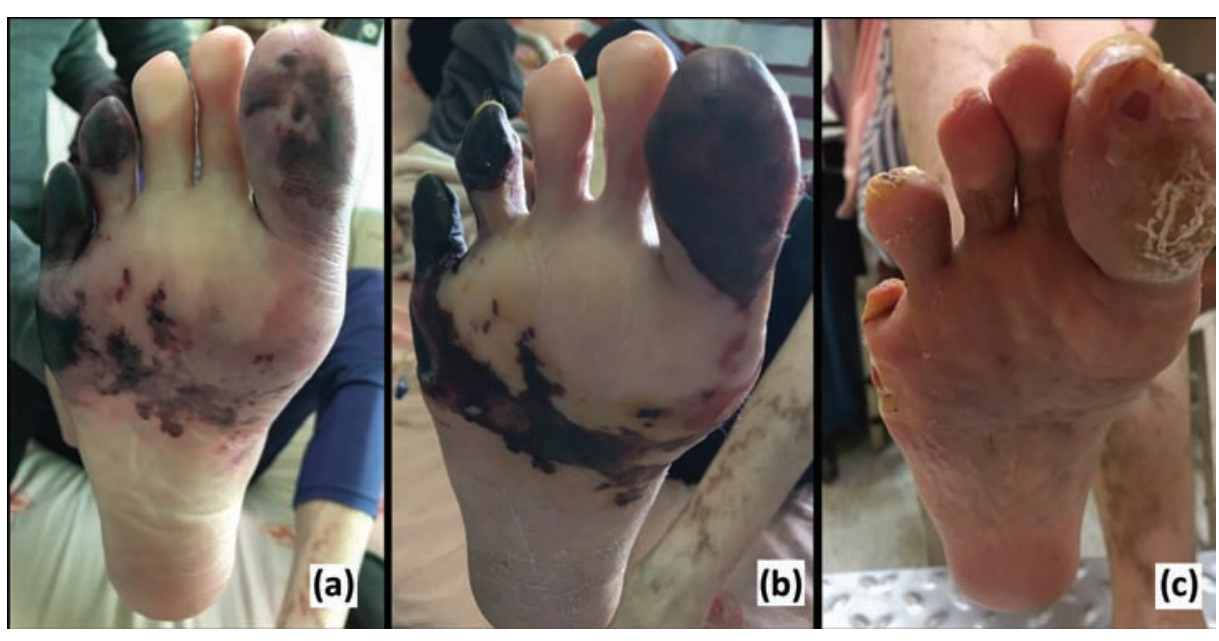

Fig 1. (a), (b) Progressive and severe Raynaud's phenomenon with severe ischemic gangrene change in the toes. (c) After aggressive treatment, auto-amputation occurred only partially, over the right 5 th toe.
Sjögren's syndrome is a chronic autoimmune exocrinopathy that can attack multiple organs, such as the pulmonary, renal, neurological and vascular systems. ${ }^{1}$ Raynaud's phenomenon is a clinical manifestation in which artery spasms cause reduced blood flow with paleness, cyanosis and even gangrenous change. ${ }^{2}$

A 47-year-old female non-smoker presented with rapid and progressive interchangeable paleness and cyanosis over the toes (Fig 1 $a$ and b) for 3 weeks. She also suffered from dry eye, xerophthalmia, numbness, and pinprick sensation over the bilateral lower limbs. Laboratory studies were positive for antinuclear antibodies (1:5120, speckle pattern) and a high titre of rheumatoid factor at $114 \mathrm{IU} / \mathrm{mL}$. Anti-citrullinated protein antibody, anti-Scl70-antibody, anti-Smith-antibody, anti-ribonucleoprotein-antibody and anti-ds-DNA-antibody tests were all negative. After further investigation, Sjögren's syndrome was impressed with positive anti-Ro-antibodies (more than $240 \mathrm{U} / \mathrm{mL}$ ), lacrimal gland function was reduced by Schirmer's test and sialoscintigraphy showed

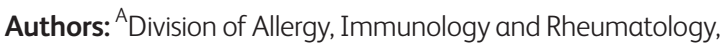

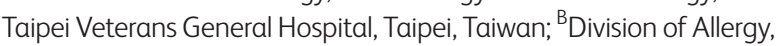
Immunology and Rheumatology, Taipei Veterans General Hospital, Taipei, Taiwan; Division of Allergy, Immunology and Rheumatology, Taipei Medical University, Taipei, Taiwan; School of Medicine, National Yang-Ming University, Taipei, Taiwan; 'Division of Allergy, Immunology and Rheumatology, Taipei Veterans General Hospital, Taipei, Taiwain; School of Medicine, National Yang-Ming University, Taipei, Taiwan dysfunction of salivary secretion. ${ }^{1}$ Tests for hepatitis C virus, human immunodeficiency virus, antiphospholipid antibodies and cryoglobulin were all normal. Oral nifedipine, sildenafil, cilostazol, clopidogrel, intravenous prostaglandin injection, hyperbaric oxygen therapy and epidural lumbar sympathetic nerve block (medical sympathectomy) halted this severe ischemic process related to progressive toes' gangrene change. After aggressive treatment, only the right 5 th toe was partially auto-amputated (Fig 1c).

\section{Consent}

Consent was obtained from the patient for publication of the clinical details and images in this article.

\section{References}

1 Shiboski CH, Shiboski SC, Seror R et al. International Sjögren's syndrome criteria working group. 2016 American College of Rheumatology/European League Against Rheumatism classification criteria for primary Sjögren's syndrome: a consensus and datadriven methodology involving three international patient cohorts. Ann Rheum Dis 2017;76:9-16.

2 Wigley FM, Flavahan NA. Raynaud's phenomenon. N Engl J Med 2016;375:556-65.

Address for correspondence: Dr Hsien-Tzung Liao, Division of Allergy, Immunology and Rheumatology, Department of Internal Medicine, Taipei Veterans General Hospital, No. 201, Sec.2, Shih-Pai Rd., Taipei 11217, Taiwan.

Email: htliao@vghtpe.gov.tw 